\title{
Introducing implementation dependent behavior into integrated product architecture clustering
}

\author{
David Williamsson ${ }^{1}$, Ulf Sellgren ${ }^{2}$ \\ ${ }^{1}$ Scania CV AB \& KTH Royal Institute of Technology \\ ${ }^{2} \mathrm{KTH}$ Royal Institute of Technology
}

\begin{abstract}
We propose a new extended version of the previously introduced Integrated Modularization Methodology (IMM) that integrates technical complexity and business strategic concerns when clustering the architecture. The extended IMM (eIMM) adds physical interference and implementation dependent behavior into product architecture clustering. A presently developed battery electric truck is used as a test bench for studying if and how the product architecture DSM and eIMM approach may enable us to identify module candidates that are reasonable trade-offs between technical complexity, business strategies and physical interference. The presented case study indicates that eIMM is able to propose a modular product architecture without conflicting business strategies or intra-modular physical interferences, as well as reasonable module candidates from a technical complexity point of view.
\end{abstract}

Keywords: Product Architecting, Integrated Modularization, DSM, MFD, ASM.

\section{Introduction}

Ulrich (1995) defines product architecture as "the scheme by which the function of a product is allocated to physical components", and more specifically "(1) the arrangement of functional elements; (2) the mapping from functional elements to physical components; (3) the specification of the interfaces among interacting physical components". Product architecture can be categorized as being modular, integral or hybrid based on the type of mapping between functional elements and physical components (Hölttä-Otto, 2005).

Products with a modular architecture are configured from predesigned modules. A module is a function carrier, with well-defined and standardized interfaces with other modules, that is configured for company-specific reasons, i.e. it supports a company-specific business strategy (Erixon, 1998). The term module variant refers to a physical incarnation of a module with a certain performance level or appearance. A modular system can be defined as the collection of module variants by which all unique products in the product family can be configured (Börjesson, 2014).

There are three main and also complementary approaches to define modularity; Heuristics, Modular Function Deployment (MFD), and Design Structure Matrix (DSM), e.g. (HölttäOtto, 2005).

Heuristics, as proposed by Stone et al. (2000), refers to rules of thumb that will likely give good results. In (Hölttä-Otto, 2005), two main categories are investigated: modules dictated by the patterns of flow (matter, energy, and information) between functional blocks, and 
Part II: Product Architecture Design

patterns of commonality/variety in a family of products. Stone et al (2000) categorized flow as being either dominant, conversion-transmission, or branching-combining. Börjesson (2012) found dominant flow to be the most usable heuristic method in most industrial cases. Heuristic methods are highly repeatable (Hölttä-Otto, 2005), but do not consider strategic objectives (Blackenfeldt, 2001).

The MFD methodology (Erixon, 1998) is a five-step approach for translating customer requirements into a modular architecture, while focusing on the company-specific strategic objectives, represented as a Module Indication Matrix (MIM) of twelve predefined generic Module Drivers (MD:s). The MIM is an interdomain matrix that relates the components and the twelve MD:s. MFD does not explicitly address technical complexity.

The main focus of $D S M$-based modularization approaches is to minimize technical complexity by clustering the DSM in a way that minimizes the technical interactions between clusters of components, i.e. complex interactions are grouped within clusters. The term cluster refers to a module candidate. Pimmler \& Eppinger (1994) proposed four generic relation types to represent the interactions between pairs of technical solutions or functions in a Product Architecture DSM (paDSM) (Eppinger \& Browning, 2012a). These relation types are spatial relations and flow of matter, information and energy. Relation weights, also known as interaction strengths, can be used to represent their relative importance. In order to distinguish desirable from undesirable relations, positive and negative values could be used, where a negative value would indicate an undesirable/harmful interaction. In (Eppinger \& Browning, 2012a) a car climate control system is represented as a DSM with positive and negative interaction values. Clustering resulted in undesirable/harmful relations between multiple components within clusters, i.e. module candidates with potential intra-modular component interference. The result of paDSM clustering often depends on the relative weights of the different types of component relations. To compare clustering results based on different relational weight combinations, a Cluster Match Matrix (CMM) is proposed by Williamsson et al. (2018).

In an attempt to balance technical complexity and company-specific business strategies, Williamsson and Sellgren (2016) introduced the Integrated Modularization Methodology (IMM). The core of IMM is to integrate company-specific module drivers (represented by the MIM) with a paDSM into a strategically adapted DSM (saDSM), which is the clustering input. Williamsson et al. (2018) confirmed that the IMM methodology can identify and propose reasonable module candidates, from both product complexity and company specific strategy points of view.

Product architecting involves product module identification and product layout design (Dieter et al., 2013a). This is a highly iterative process that benefit from representations that represent change. De Weck (2007) introduced Component-Based $\triangle D S M$ and ChangeDSM to represent and manage existing or future changes in complex products. A $\triangle D S M$ represents the difference between an original and a changed product. The Change-DSM contains the change propagation paths, i.e. how a change propagates from one component to another.

Making a rough spatial layout of the product enables analyses of potential spatial, thermal, or electrical interferences between components within module candidates. In the Affordance Based Design theory (Maier and Fadel, 2008), an Affordance Structure Matrix (ASM) may be used in the conceptual design stage to augment the DSM, by representing the relations between system components and affordances. An affordance is what one 


\section{Williamsson, U. Sellgren}

system provides to another system (or part of a system, e.g. a component). Unlike functions, affordances are form-dependent. With the main purpose to identify components with an improvement potential, Maier et al. (2008) modified the original ASM, with relations represented as existing or not, by specializing the relation types as being helpful $(+)$, neutral ( ) or harmful (-). ASM may thus be used to represent harmful or undesirable interferences between components.

The purpose of the research presented in this paper is to study if the existing functionmeans oriented IMM clustering method, that targets strategic and technical complexity issues in the architecting systems engineering stage, can be extended to also include unwanted side-effects caused by harmful interferences. Three specific research questions are addressed in this paper:

- Does paDSM clustering with negative relation weights, representing undesirable/harmful relations, propose clusters without physical interference?

- Could an affordance based design approach augment existing IMM clustering by taking physical interferences into consideration?

- Could the extended IMM approach enable effects from technical complexity, strategic aspects and physical interferences to be analyzed in any combination?

The research is based on a case study of a battery-electric powerline of a heavy duty truck. The case is presented in chapter two, analyzed and discussed in chapters three and four. The work is concluded in chapter five and further work is proposed in chapter six.

\section{Case study}

The presented architectural study was performed as action research at the heavy truck manufacturer Scania, which is part of TRATON SE (owned by Volkswagen AG), and at KTH Royal Institute of Technology in Stockholm, Sweden.

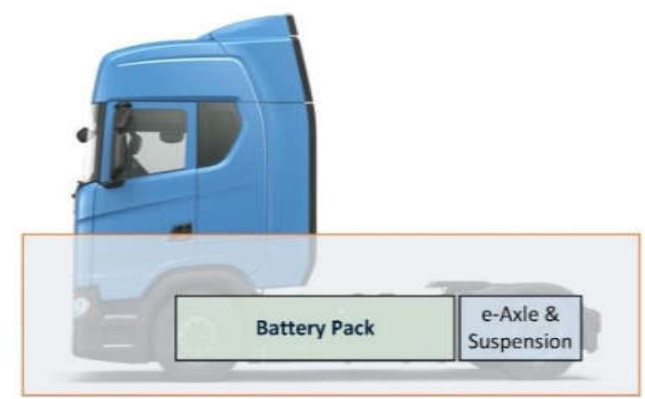

Figure 1. Cutaway illustration of a Scania BEV concept layout.

The case study focused on the main parts of a conceptual powertrain, suspension and braking system of a future Battery Electric Vehicle (BEV) product variant, see Figure 1. Evaluating the complete modular truck system is beyond the scope of this paper but it will be addressed in future publications. Hence, one product variant (i.e. one configuration) was analyzed in the presented study. The BEV architecture is partly developed in-house by Scania to be a future module in the modular truck system. The powertrain contains 
Part II: Product Architecture Design

synergistic configurations of mechanical, electrical and software components that are constituents of an automated and/or semi-autonomous system. The architectural investigation required an initial analysis of the mechanical, electrical and embedded software subsystems.

\subsection{The studied product architecture}

The core of Scania's modularization principle is carefully balanced module variants configured from a limited number of components, with standardized interfaces, that can be combined to satisfy individual customer needs.

After interviewing domain experts and investigating the logical structure of the electrical and software components, the studied conceptual BEV architecture under development was represented with a Component Architecture Diagram (CAD) (Williamsson et al., 2018), see Figure 2. To limit the number of components, all screws and other small parts, were not considered in the analysis. The interactions of the targeted 63 components were represented in the diagram, which visualizes the components, the principal technical function flows and spatial relations. In Figure 2, black lines indicate spatial relations, green energy flows, blue material transfer and orange information flows. The CAD is only representing a first rough spatial layout of the studied product.



Figure 2. The component architecture diagram of the studied architecture.

The main difference between the investigated BEV concept and the current powerline is the replacement of the Internal Combustion Engine (ICE) and the mechanical transmission by a battery pack and a new electric rear axle, called e-axle. The e-axle implements the propulsion, braking and transmission related functions. Since the ICE contains many supporting functions, e.g. generate heat to the cabin, these functions must be implemented by a large amount of new technical solutions in the BEV. A simple approach, in terms of risk and cost, is to simply add an electric motor to a traditional mechanical axle, thus reducing the need for many new components and interactions. However, this is typically not an optimal solution in terms of energy efficiency, volume utilization and cost. Due to the relatively low energy density in the batteries, the operating range of a BEV is highly 


\section{Williamsson, U. Sellgren}

dependent on the volume and weight of the battery pack. Therefore, it is important to minimize the volume and weight of the e-axle to allow for a large size battery pack.

When making larger changes of an existing generic product architecture and integrating multiple electrical components into the rear axle, it is crucial to analyze undesirable and potentially harmful effects in the system as early as possible in the product architecting stage. For example, some components may be sensitive to vibrations, heat and/or electromagnetic fields and should preferably not be clustered with components generating or transmitting vibrations, heat and/or magnetic fields, respectively.

\section{Analysis method and results}

The modeled BEV powertrain architecture was used as a test bench for studying if and how the DSM and an ASM-extended IMM (eIMM) approach may enable identification of module candidates that are reasonable trade-offs between technical complexity, business strategies and physical interferences.
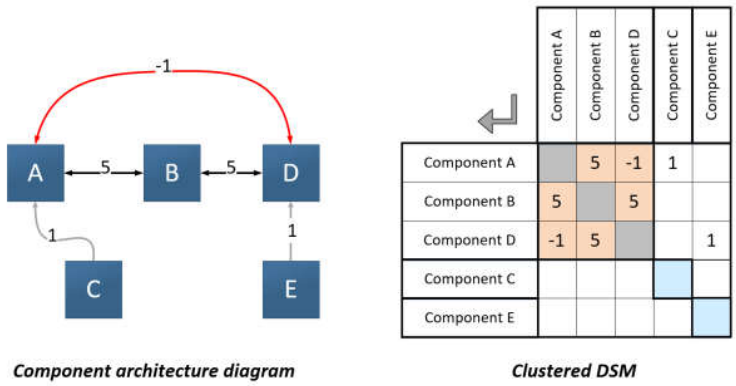

Figure 3. Example of a CAD and clustered DSM with negative relation weights.

As earlier defined, one way to represent components with undesirable and harmful relations in a DSM is to assign negative relation weights. An example of a clustered paDSM with negative relation weights is presented in figure 3 . In this example, components $A, B$ and $D$ were clustered together, resulting in a module candidate with component interference. Interference between module candidates is often far easier to manage compared to interference within module candidates, simply because interference frequently can be resolved by physical separation, i.e. positioning module candidates in an alternative product configuration. The presented example highlights some limitations of DSM clustering with negative relation weights.

\subsection{The proposed eIMM architectural analysis method}

The studied architecture was represented as a paDSM and a layout adapted DSM (laDSM). ASM-extended IMM (eIMM) clustering was performed of the laDSM. The clustering analyses were performed with the clustering algorithm IGTA++ (Börjesson \& Sellgren, 2013). The four types of interactions in the DSM were initially assumed to have equal importance (weight) in the off-diagonal matrix cells. The strategies addressed were the MD:s in the MFD methodology. 


\section{Part II: Product Architecture Design}

The starting point of any IMM-based analysis is a product architecture representation, i.e. a paDSM. The relations between corporate strategies, as represented by the MD:s, and the technical solutions, i.e. the components in the paDSM, are represented with the Module Indication Matrix (MIM) in the MFD method. One of the main purposes of an MIM is to identify strategically conflicting MD:s. According to MFD, strategically conflicting module drivers should not be clustered together. In IMM, the MIM (see upper left part of Figure 4) is represented by a strategy transfer DSM (see lower left matrix in Figure 4), with all conflicting module drivers represented by a minus sign. By only operating with the strategy transfer DSM on the paDSM, we get a strategically adapted DSM (saDSM). By operating with the strategy transfer DSM and affordance transfer DSM on the Product Architecture DSM, we get the layout adapted DSM (laDSM).



Figure 4. An extension of the integrated DSM-based product architecting method IMM.

The components which always are included in the investigated architecture, independent of configuration, i.e. the platform modules, were treated as common unit components, the green components in Figure 2. Furthermore, components which must be developed due to technology shifts, were treated as technology evolution components, shown as red components in Figure 2. The remaining components were treated as different specification and/or carryover components (which are non-conflicting module drivers).

The working hypothesis is that the paDSM and SaDSM do not contain any (or limited) physical interference information and is therefore not capable of enabling physical interference analyses in the later part of the architectural stage. The core of the new ASMextended version of the IMM (eIMM) is an ASM representing if component-affordance relations are helpful $(+)$, harmful (-), or neutral ( ). By adding the number of affordances with which each component has a helpful, harmful or neutral relationship, a total score can be calculated. This score gives a rough indication of the total harm or good each component is causing, as well as the potential for future improvements. In our proposed eIMM approach, the score is mainly used to identify components which may be harmful to components having conflicting affordances, i.e. components having physical interference which should not be clustered together. The "roof" of the ASM in our approach is therefore only used to represent components having conflicting affordances, indicated with minus 


\section{Williamsson, U. Sellgren}

signs. This is a similar approach as how conflicting module drivers are treated. Hence, this extended approach aims to bring physical interference and implementation dependent behavior into the clustering operation.

In the present case study, components which are sensitive to vibrations, i.e. the components marked as purple in Figure 2, were treated as having conflicting affordances to all remaining vibration emitting or vibration resistant components.

\subsection{Architectural analysis of the BEV architecture}

The modular view of the studied BEV architecture is represented as a Component Cluster Diagram (CCD), see (Williamsson et al., 2018), which is a simplification of the CAD, since it represents the modular clusters without interactions. The relations were assumed to be of equal importance, i.e. equal weight, in the initial clustering analysis. This assumption enables a comparison of how an assumed increasing importance of the information flow in future generations would affect the clustering result. The values used for the relation weights were 1 (functional dependency) or 2 (strong dependency). Convergence of each clustering result was found after 3000 iterations with the IGTA++ clustering algorithm in MATLAB.

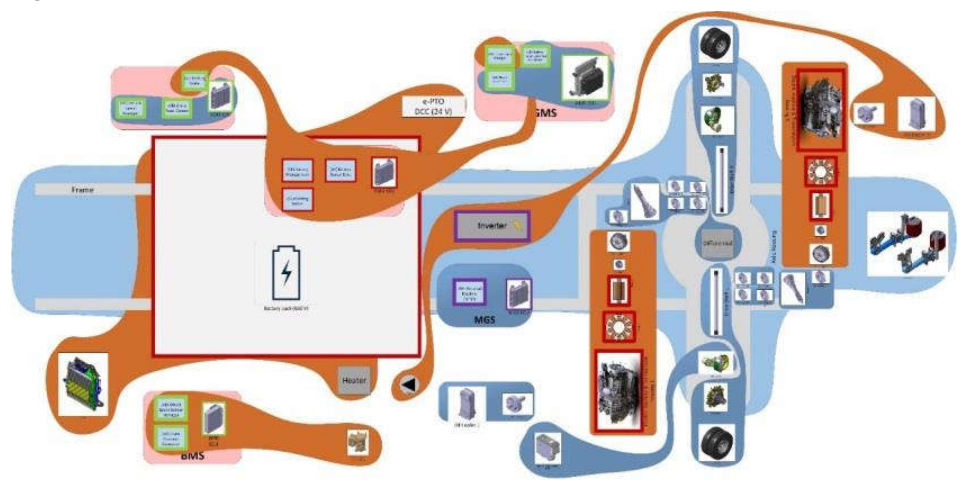

Figure 5. DSM clustered modular architecture (equal relation weights). (6 conflicting clusters).

The results from the DSM and ASM-extended IMM clustering analyses are presented in figures $5-7$, where the module candidates are visualized as blue and orange shapes (clusters). A blue shape indicates that there is no conflict within the cluster. An orange shape indicates that the cluster contains components with conflicting MD:s or affordances, i.e. in this case common unit and different specification components, or technology evolution and carryover, or vibration emitting and vibration sensitive.

DSM clustering with equally important relation weights gave several conflicts within the clusters. These clusters with conflicts are marked with an orange color in Figure 5. After analyzing the DSM with equal relation weights, the importance of the information flows was determined to be two times higher than the other three types of relations. The result of this analysis is presented in figure 6 .

To analyze the influence on module clustering from both strategic and physical interference reasons, clustering of an laDSM was performed with the proposed eIMM approach. As seen in figure 7, the resulting clusters are somewhat different compared to the two previous 
Part II: Product Architecture Design

DSM-based analyses, though several clusters are identical. However, as seen in figure 7, there are no conflicting MD:s or affordances are in the clusters proposed by eIMM.

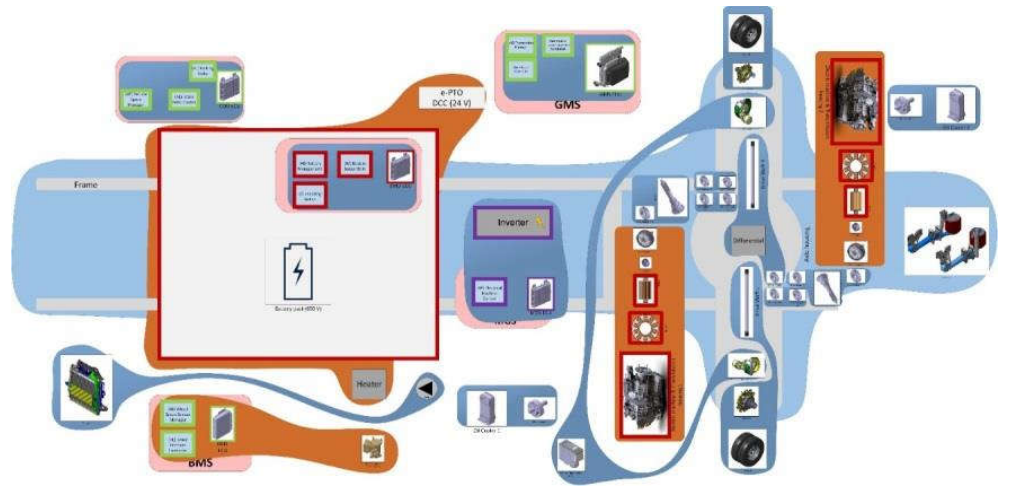

Figure 6. DSM clustered modular architecture, strong dependency of information flow. (4 conflicting clusters).

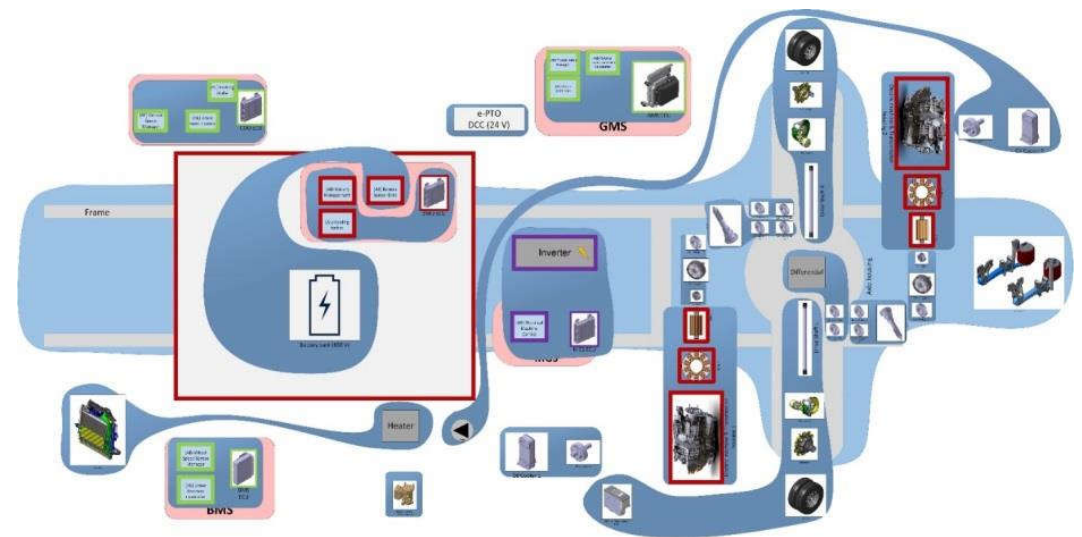

Figure 7. ASM-extended IMM clustered modular architecture (equal relation weights). (0 conflicting clusters).

\section{Discussion}

When making large architectural changes, (or developing a completely new product), it is highly important to use a robust product architecting methodology which supports the highly complex task with a structured method and efficient tools, otherwise important aspects may not be treated properly.

It is important to analyze not only function-means aspects but also undesirable and harmful effects in the product architecting stage. One approach to identify harmful or undesirable effects in a system is to model desirable and undesirable relations between components in the DSM. In order to distinguish desirable from undesirable relations, positive and negative values can be used. However, this approach does not guarantee that components with 


\section{Williamsson, U. Sellgren}

negative relations are not clustered together, i.e. the clusters may contain physical/affordance conflicts. Hence, a new methodology is clearly needed.

IMM aims to augment a traditional DSM clustering approach by taking strategic aspects, and now also physical interference into consideration during the clustering stage. The core of the new eIMM methodology is a layout adapted DSM (laDSM), which is a DSM that integrates a paDSM with a MIM from the MFD methodology and an ASM. The DSM approach, which targets technical complexity, does not contain any strategic and only limited physical interference information and is therefore not capable of handling these aspects in the clustering stage. This limitation of paDSM clustering has been illustrated and confirmed in this study.

The eIMM is able to treat multiple matrices (representing technical complexity, strategic aspects and physical interference), which can be configured depending on the purpose of each analysis. Design knowledge generally increases during the development of a product. The eIMM approach allows the user of the method to add aspects as new matrices. The reason for not integrating all matrices into one single DSM is also due to analysis advantages. Separation makes it possible to, e.g., investigate if and how the clusters are changed when business strategies and/or physical interference are introduced. These are important features of the scalable and flexible eIMM approach.

The presented case study shows that eIMM proposes a modular product architecture without conflicting MDs or affordances, as well as reasonable module candidates from a technical complexity point of view. As seen in the case study, the proposed clusters tend to be changed if the weight of e.g. information flow is assigned with a relatively high importance compared with other types of relations. Moreover, the proposed clusters also tend to be changed if strategies (MD:s) or affordances are introduced, see the DSM and eIMM clustering results in the case study.

It should be noted that even though the eIMM aims to cluster components in such a way that conflicting affordances are avoided, i.e. physical interference within clusters, undesirable and harmful effects between clusters still needs to be resolved in the trial layout or (if required) in the detail design phase. The case study presented in this paper includes a rough spatial layout (see the $\mathrm{CAD}$ in figure 2). Hence, the presented results are important knowledge input to the future trial layout phase.

\section{Conclusions}

The findings from the presented case study can thus be summarized as follows:

- Representing undesirable and harmful relations in a product architecture DSM by assigning negative relation weights does not guarantee clusters with no physical interference.

- The presented case study indicates that the proposed eIMM is capable of identifying and proposing module candidates without conflicting MDs or affordances, as well as reasonable module candidates from a technical complexity point of view. 
Part II: Product Architecture Design

- $\quad$ The eIMM is modular, allowing the effects from technical complexity, strategic aspects and physical interference to be analyzed independently or combined depending on purpose.

\section{Further work}

The long term aim of the presented research is to develop a robust, agile and efficient modular architecting methodology. To verify, generalize, and improve the proposed eIMM approach and its applicability in the systems engineering process, a larger range of products and development cases must be analyzed. It is crucial to investigate the reasons, i.e. performance, reliability, safety, cost and other concerns, for defining proper weights, and how they affect clustering results.

The next step in the presented case study will be to create a trial layout, i.e. position the module candidates in a possible physical product configuration. To improve the complete modular system, many more product variants must also be analyzed. One important aspect to consider when optimizing the modular system is to identify possible modules which can be shared across multiple platforms.

\section{Acknowledgements}

The financial support from SCANIA CV AB, as well as the open discussions with domain experts at SCANIA and TRATON are gratefully acknowledged.

\section{References}

Blackenfelt, M. (2001) Managing complexity by product modularization. Doc. Thesis, Dept. of Machine Design, KTH Royal Institute of Technology, Stockholm, Sweden.

Börjesson, F. (2012) Approaches to modularity, Licentiate Thesis, Dept. of Machine Design, KTH Royal Institute of Technology, Stockholm, Sweden.

Börjesson, F., Sellgren, U. (2013) Fast Hybrid Genetic Algorithm for Clustering Design Structure Matrix. ASME Design Eng. Tech. Conf., Portland, Paper number DETC2013-12041

Börjesson, F. (2014) Product platform design - architecting methods and tools, Doc. Thesis, Dept. of Machine Design, KTH Royal Institute of Technology, Stockholm, Sweden.

Dieter, E. George \& Schmidt, C. Linda, (2013a) Engineering Design, 5th edition, New York, NY, McGraw-Hill.

Eppinger, S.D. \& Browning, T.R. (2012a) Design Structure Matrix Methods and Applications. Cambridge, Massachusetts: The MIT Press.

Erixon, G. (1998) Modular function deployment: a method for product modularization, Doc. Thesis, Dept. of Manuf. Sys., KTH Royal Institute of Technology, Stockholm, Sweden.

Hölttä-Otto, K. (2005) Modular Product Platform Design, Doc. Thesis, Department of Mechanical Engineering, Machine Design, Helsinki University of Technology, Helsinki, Finland.

Maier, J.R., Sandel, J. \& Fadel, G.M. (2008) Extending The Affordance Structure Matrix - Mapping Design Structure and Requirements to Behavior. $10^{\text {th }}$ International Design Structure Matrix Conference, Stockholm, Sweden.

Maier, J.R., \& Fadel, G.M. (2008) Affordance based design - a relational theory for design. SpringerVerlag, London. DOI 10.1007/s00163-008-0060-3. 


\section{Williamsson, U. Sellgren}

Pimmler T.U., Eppinger S.D. (1994) Integration Analysis of Product Decompositions, ASME Design Tech. Conf., $6^{\text {th }}$ International Conference on design Theory and Methodology, DTM '94, Minneapolis, USA.

Stone, R.B., Wood, K.L., Crawford, R.H. (2000) A heuristic method for identifying modules in product architectures. Des. Studies, 21 (1), 5-31.

Ulrich, K. (1995) The role of product architecture in the manufacturing firm. Research Policy 24(3):419-440, DOI 10.1016/0048-7333(94)00775-3, ISSN 0048-7333.

Williamsson, D. and Sellgren, U. (2016) An approach to integrated modularization, 26th CIRP Design Conf., Stockholm, Sweden.

Williamsson, D. and Sellgren, U., Söderberg, U. (2018) A hunt for the hidden reasons behind a product architecture. 20th Int. Dependency and Structure Modeling Conf., DSM 2019, October 15-17, Trieste, Italy.

Contact: D. Williamsson, Scania CV AB, R\&D, Product Description Methodology, Transmissionsvägen 2, 151 48, Södertälje, Sweden, david.williamsson@scania.com 